\title{
EMPRENDIMIENTO E INNOVACIÓN: AGENTES POTENCIADORES DE LA EMPRESA FAMILIAR ${ }^{1}$
}

\author{
Entrepreneurship and innovation: enhancing agents of family business / \\ Empreendimento e inovação: agentes potenciadores da empresa familiar
}

\author{
Flor Salazar²; Jorge González ${ }^{3}$; Paola Sánchez ${ }^{4}$ y Jhonnathan Sanmartin ${ }^{5}$
}

\begin{abstract}
RESUMO
O presente trabalho tem como propósito, apresentar uma reflexão teórica sobre o emprendimento e a inovação, como agentes potenciadores da empresa familiar. Insere-se num paradigma qualitativo, apoiado numa revisão documental, especificamente textos e artigos científicos, derivados de base de dados especializada, de autores de prestígio na área, tais como: Navarro e Villegas (2016), Vesga (2017), Rockefeller (2004), entre outros. Uma vez realizada a revisão bibliográfica, importa referir que os resultados mostram que existe uma necessidade de fortalecer a inovação, para promover a sustentabilidade desse tipo de empresas e, desta forma, deve reunir-se esforços das políticas públicas definidas para o fomento da actividade empreendedora. Estas, por sua vez, provêm dos recursos financeiros que devem ser aproveitados para o desenvolvimento, tanto das empresas familiares, como das comunidades nas quais se inserem. Deve existir uma cultura empreendedora apoiada na inovação, que permita potenciar a economia familiar e a sociedade, em geral.
\end{abstract}

Palavras Chave: Empreendimento, Inovação, Empresa Familiar.

Recebido: Outubro $2018 \quad$ Aceitado: Dezembro 2018

\section{RESUMEN}

El presente trabajo tiene como propósito realizar una reflexión teórica sobre el emprendimiento y la innovación como agentes potenciadores de la empresa familiar. Se inserta en un paradigma cualitativo, apoyado en una revisión documental, especificamente textos, y artículos científicos derivados de base de datos

\footnotetext{
1 Este trabajo muestra resultados parciales del proyecto de Vinculación con la sociedad: "Desarrollo de capacidades creativas, mejoramiento y gestión de emprendimiento en las Asociaciones agrícolas del Cantón La Troncal". Financiado por la Universidad Católica de Cuenca

2 Ingeniera empresarial, Máster en Auditoría Integral, Máster en Docencia Superior. Docente e investigador de la Universidad Católica de Cuenca, Ecuador. Correo electrónico: fisalazarv@ucacue.edu.ec

${ }^{3}$ Economista, Magíster en Auditoría Integral. Docente e investigador de la Universidad Católica de Cuenca, Ecuador. Correo electrónico: jgonzalezu@ucacue.edu.ec

4 Estudiante. En Ing. empresarial. de la Universidad Católica de Cuenca, Ecuador. Correo electrónico: pmsanchezs93@est.ucacue.edu.ec

${ }^{5}$ Abogado, libre ejercicio profesional. Correo electrónico: ihonnathan71@live.com
} 
especializada de reconocidos autores en el área, tales como: Navarro y Villegas (2016), Vesga (2017), Rockefeller (2004), entre otros. Los resultados muestran luego de la revisión bibliográfica, que existe una necesidad de fortalecer la innovación para promover la sostenibilidad de este tipo de empresas; asimismo, se debe reorientar esfuerzos en funcion de las políticas públicas definidas hacia el fomento de la actividad emprendedora; estas proveen los recursos financieros que deben ser aprovechados para el desarrollo tanto de las empresas familiares como de las comunidades en las cuales se insertan. Debe existir una cultura emprendedora apoyada en la innovación que permita potenciar la economia familiar y de la sociedad en general.

Palabras clave: Emprendimiento, Innovación, Empresa Familiar.

\section{ABSTRACT}

The purpose of this paper is to carry out a theoretical reflection on entrepreneurship and innovation as empowering agents of the family business. It is inserted in a qualitative paradigm, supported by a documentary review, specifically texts, and scientific articles derived from a specialized database of recognized authors in the area, such as: Navarro and Villegas (2016), Vesga (2017), Rockefeller (2004), among others. The results show, after the literature review, that there is a need to strengthen innovation to promote the sustainability of this type of companies; likewise, efforts must be reoriented in function of the public policies defined towards the promotion of entrepreneurial activity; these provide the financial resources that should be used for the development of both family businesses and the communities in which they are inserted. There must be an entrepreneurial culture based on innovation that allows the strengthening of the family economy and of society in general.

Keywords: Entrepreneurship, Innovation, Family Business.

\section{INTRODUCCIÓN}

El crecimiento económico de los países y sus regiones ha estado marcado en los últimos tiempos por el Emprendimiento y la Innovación agentes potenciadores de la economía y la generación de empresas. En este sentido Rockefeller (2004), define el emprendimiento como la iniciativa de uno a varios individuos con amplia capacidad y proyectividad en el campo del proceso empresarial y social capaces de hacer transformaciones y obtener resultados exitosos y sobresalientes.

De acuerdo a este autor, se afirma que dentro de esta iniciativa se encuentran inmersas una serie de cualidades propias del individuo como: capacidades, aptitudes, motivación, conocimientos y habilidades que han contribuido de forma 
relevante a la constitución de organizaciones denominadas empresas familiares, a nivel nacional e internacional.

Aunado a lo anterior, la capacidad y proyectividad del individuo para emprender cualquier tipo de proyecto, está condicionado por el componente innovativo, dado que, la innovación es una de las causas del desarrollo económico, considerado un proceso de transformación económica, social y cultural, se refiere a la introducción de nuevos bienes y servicios en el mercado, el surgimiento de nuevos métodos de producción y transporte, la consecución de la apertura de un nuevo mercado, la generación de una nueva fuente de oferta de materias primas y el cambio en la organización en su proceso de gestión (Schumpeter, 1942).

En este sentido, para los fines del presente trabajo consideramos la relevancia que adquiere la interrelación, interacción, y la sinergia existente entre el innovar y emprender para la creación de una diversidad de nuevas empresas, para el propósito de nuestra investigación hablamos específicamente de las empresas de tipo familiar, en este tipo de iniciativas, son consideradas un componente esencial para hacer los procesos más eficientes en cuanto a la producción de bienes y servicios, y la forma de gestionarlos.

Dentro de este contexto, las empresas familiares son concebidas por Rodríguez y Chauca (2013) y Leach (1993), como aquélla que está influenciada por una familia o por un vínculo familiar. La familia como entidad puede controlar efectivamente las operaciones de la empresa, porque posee más del $50 \%$ de las acciones o porque miembros de la familia ocupan importantes posiciones en la dirección.

De acuerdo a lo expresado por los autores, las empresas familiares están conformadas básicamente por el elemento consanguíneo de un grupo de personas dispuestas a ejecutar un proyecto de cualquier índole, estas organizaciones emergen en gran parte de la capacidad que tienen los miembros de las familias que forman parte de sus comunidades, de emprender o ejecutar una idea innovadora que les permita su desarrollo a nivel personal y económico, son generadoras de un gran número de fuente de trabajos e ingresos que permiten el desarrollo de sus comunidades y se constituyen en microempresas familiares. 
Esta afirmación se hacen de acuerdo a Rodríguez y Chauca (2013) y Poza (2004), quienes expresan, que aproximadamente el $90 \%$ de las empresas en el mundo son familiares; que tienen una gran aportación al empleo y al producto interno bruto (PIB) mundial.

Cabe destacar, que para que estas empresas familiares sean capaces de generar rentabilidad y sostenibilidad deben estar apoyadas en políticas públicas que fomenten el crecimiento de este tipo de organizaciones y les faciliten el apoyo financiero necesario por parte del Estado mediante el otorgamiento de créditos que coadyuven a la puesta en marcha de la empresa familiar, que han demostrado en el transcurrir de los últimos años el aporte relevante que representan para dinamizar la economía y acelerar el empoderamiento de los ciudadanos para su propio beneficio y el de la sociedad de la cual forman parte.

Por todo lo anteriormente expresado, el presente trabajo de investigación, tiene como propósito realizar una reflexión teórica sobre el emprendimiento y la innovación como agentes potenciadores para la empresa familiar.

De igual forma, constituye una muestra de los resultados parciales del proyecto de investigación titulado: "Desarrollo de capacidades creativas, mejoramiento y gestión de emprendimiento en las Asociaciones agrícolas del Cantón La Troncal". Financiado por la Universidad Católica de Cuenca.

\section{ASPECTOS METODOLÓGICOS}

El presente estudio se fundamentó en el método cualitativo, el cual trata de descubrir y construir los objetos de conocimientos dividiendo la realidad en sus partes más elementales. Se parcializa y segmenta el objeto de lo más simple a lo más complejo, bajo un enfoque epistemológico cualitativo, el cual consiste en utilizar métodos de recolección de datos sin medición numérica (descripciones y las observaciones), en el cual el énfasis no está en la medición de las variables involucradas en dicho fenómeno, sino en entenderlo, comprendiendo la conducta humana desde el propio marco de referencia de quien actúa y fundamentado en la realidad, orientado a los descubrimientos, exploratorio, expansionista, descriptivo e inductivo (Hernández y col., 2006). 
Se plantea en el artículo de investigación una reflexión que parte de la revisión de diversas fuentes documentales, con abordajes teóricos de reconocidos autores sobre emprendimiento e innovación como: Navarro y Villegas (2016), Vesga (2017), Rockefeller (2004). De igual forma, Belausteguigoitia (2007), Poza (2004), Leach (1993), entre otros, soportan la tesis sobre empresa familiar. A fin de establecer posturas relevantes para el favorecimiento de propuestas de investigación que coadyuven en el fortalecimiento y desarrollo del emprendimiento y la innovación como agentes potenciadores para la creación de empresas familiares exitosas y sostenibles.

\section{LA INNOVACIÓN: FACTOR CLAVE PARA EL DESARROLLO DEL EMPRENDIMIENTO}

Algunas definiciones necesarias:

La innovación constituye el principal componente para el aumento de la productividad de los países, considerado según Schumpeter (1942) como una de las causas del desarrollo económico, es un proceso de transformación económica, social y cultural, trata de la introducción de nuevos bienes y servicios en el mercado, el surgimiento de nuevos métodos de producción y transporte, la consecución de la apertura de un nuevo mercado, la generación de una nueva fuente de oferta de materias primas y el cambio en la organización en su proceso de gestión.

Dentro de esta perspectiva, Vesga (2017) considera la innovación como un proceso deliberado que permite el desarrollo de nuevos productos, servicios y modelos de negocios en las organizaciones, generando resultados concretos, medibles, y está asociado al desarrollo de un espíritu emprendedor en los individuos, es decir, a una actitud hacia el trabajo que se caracteriza por la permanente búsqueda de oportunidades y por la capacidad para articular recursos humanos y físicos con el objetivo de aprovecharlo.

Navarro y Villegas (2016), expresan la innovación es resultado de un aprendizaje dinámico en su naturaleza y fuertemente dependiente de su trayectoria, es un proceso sistemático de aprendizaje, búsqueda y exploración llevada a cabo 
por diversos actores, firmas, gobierno y universidades para la generación de nuevos productos, técnicas de producción y formas de organización; dentro de esta definición también se incluye el proceso de mejoras en productos y técnicas existentes.

Estos autores afirman, que la innovación es relevante para las organizaciones por el impacto que generan sobre su rendimiento y en especial sobre sus posibilidades de supervivencia de largo plazo. Sin embrago, la innovación está inmersa en diferentes perspectivas, desde el punto de vista macroeconómico, la innovación es uno de los principales determinantes del crecimiento económico. Los mecanismos que incentivan este proceso aún están en discusión (Navarro y Villegas, 2016)

Es posible afirmar que los principales factores que generan incentivos para una mejora continua en el proceso de innovación dependen principalmente del agente quien lo realiza, el marco institucional en el que se lleva a cabo este proceso, la estructura de mercado en la cual se realiza la innovación y las características de la división del trabajo en el proceso productivo dentro de una industria (por ejemplo, empresas que se dedican a la comercialización del producto -downstream-y otras que se dedican exclusivamente a la creación de nuevas tecnologías -upstream-) (Navarro y Villegas, 2016).

Según Navarro y Villegas (2016) siguiendo a Baldwin y Hanel (2003), la innovación puede ser entendida como un proceso continuo cuyas características cambian a lo largo del ciclo de vida del producto. El ciclo de vida del producto caracteriza el proceso de innovación en cuatro principales etapas: a) con el surgimiento de una innovación, emerge un producto nuevo y un número reducido de empresas trabajan para poder comercializarlo; b) se genera un período de ingreso y salida de empresas en el mercado del producto que surgió con la innovación, en esta etapa un gran número de empresas le hacen modificaciones al producto original y al proceso de producción; c) el ingreso de nuevas firmas se reduce, pero la salida es masiva, las empresas que quedan empiezan a cubrir la mayor parte del mercado y, por lo tanto, incrementan su tamaño; y d) en cierto punto, las empresas restantes aprovechan las economías a escala que se generan al 
producir en masa, descendiendo de esta forma en su curva de costos (Gort y Klepper, 1982).

Lo expresado, conduce a la identificación de dos tipos de procesos de innovación: la innovación en ciencia básica y la innovación de carácter comercial (Navarro y Villegas, 2016). Las innovaciones en ciencia básica son aquellas que producen un aporte fundamental al conocimiento humano. Este tipo de innovaciones son muy importantes, porque, debido a su carácter general, constituyen la base de nuevas innovaciones en diversos campos industriales, pero en principio se muestran como innovaciones embrionarias que no generarán un producto comercial directamente o un proceso productivo nuevo en el corto plazo.

Asimismo Navarro y Villegas (2016), afirman que las ideas que generan este tipo de innovaciones son fuente de grandes externalidades positivas a nivel social, por lo que la apropiación privada de las mismas no es óptima.

Las innovaciones de carácter comercial son aquellas que tienen como objetivo generar un nuevo proceso que mejore la organización y funcionamiento del proceso productivo o un nuevo producto que pueda ser introducido en el mercado. Este tipo de innovación usualmente es protegido por derechos de propiedad, lo que le otorga al agente que genera esta innovación el derecho de apropiarse de las rentas económicas y protegerse de otros agentes que quieran usar esta innovación para su propio beneficio (Navarro y Villegas, 2016).

De lo expresado por los autores, se puede afirmar que en la actualidad se ha demostrado a través de varios estudios realizados en diferentes países del mundo la relevancia de la innovación como factor clave para el desarrollo de emprendimientos, enfocados en actividades de producción de bienes y servicios, insumos y procesos.

En este sentido, se infiere que el fenómeno de la innovación cobra cada día mayor importancia y debe estar presente en toda y cada una de las actividades económicas de los países, por lo que se considera el elemento primordial para la generación de nuevos proyectos, estructuras y procesos -innovar- es la capacidad y la cualidad que tiene el ser humano proveniente del conocimiento para generar una idea o un proyecto de emprendimiento que pueda ser medible y cuantificable 
para intervenir y transformar realidades sociales, este debe ser gestionado y puesto en práctica con el fin de generar bienestar social y empresarial en las sociedades y su comunidad.

Estas afirmaciones, colocan en manifesto la vinculación entre la innovación y el emprendimiento, la complementariedad que existe entre ellos, y que han demostrado en el transcurrir de los últimos años ser el motor de impulso de las economías emergentes para la recuperación económica de las sociedades, entendiendo el emprendimiento como el acto de "Acometer y comenzar una obra, un negocio, un empeño, o proyecto especialmente si encierran dificultad o peligro" (Diccionario de la Real Academia Española, 2001).

\section{EMPRENDIMIENTO: AGENTE POTENCIADOR DE LA EMPRESA FAMILIAR}

En diversos estudios realizados desde el siglo XVIII hasta la actualidad sobre el emprendimiento, se ha demostrado, que este constituye un motor de impulso para la economía de los países, ya que viene dado por la iniciativa de uno a varios individuos con amplia capacidad y proyectividad en el campo del proceso empresarial y social capaces de hacer transformaciones y obtener resultados exitosos y sobresalientes (Rockefeller, 2004).

Según Drucker (1985), el emprendimiento es "el proceso de obtención de beneficios de nuevas combinaciones únicas y valiosas de recursos en un entorno incierto y ambiguo". No es un emprendedor innovador el que repite lo que se ha hecho ya otras veces. No es emprendedor aunque el nuevo negocio represente una nueva aventura comercial.

De igual forma, el emprendimiento es considerado por Vallmitjana (2011), como una fuerza crítica para el crecimiento económico de un país ya que supone la creación de nuevos puestos de trabajo, el aumento para el estado de los ingresos procedentes de impuestos, el incremento de las exportaciones y, en definitiva, comporta un aumento de la productividad nacional.

Aunado a lo anterior, se considera pertinente esbozar las características de la persona emprendedora, para la Corporación de Fomento de la Producción 
(CORFO, 2014) se identifica por poseer cualidades como: resiliencia, compromiso, liderazgo, búsqueda persistente de oportunidades, baja aversión al riesgo, creatividad, autonomía y motivación para sobre salir. Sin embargo, todo esto está condicionado por el contexto, es decir, condiciones del entorno compuesto por las instituciones, regulaciones y aspectos socioculturales que influyen en el nacimiento y crecimiento de las empresas que podrían facilitar o impedir la actividad emprendedora y es sobre ello donde deben actuar las políticas de emprendimiento e innovación.

\section{FACTORES QUE INHIBEN LA ACTIVIDAD EMPRENDEDORA}

Oporto (2016) afirma, el emprendimiento está condicionado por el entorno económico, la estructura productiva, el tejido empresarial, los recursos humanos, la institucionalidad, el papel de la sociedad civil, por lo que en economías basadas en recursos, con más atraso económico y con una estructura productiva estrecha, se afrontan más dificultades para convertir el espíritu emprendedor en gestión empresarial y consolidar los negocios nacientes en estables, sostenibles y con aptitud de escalamiento.

Dentro de este orden de ideas, Vallmitjana (2011), expone que hay ciertos factores que inhiben la actividad emprendedora: a) dificultad que supone planificar y entender una nueva actividad, por el simple hecho de no conocerla; b) la inercia que se instala en la propia mente del hombre de negocios; c) la tendencia humana a rechazar cualquier conducta que se desvíe de lo rutinario, incluso si es para mejorar el modo de hacer las cosas.

Según Vallmitjana (2011), existe una diversa gama de emprendimiento como: autoempleos o emprendimiento por necesidad, emprendimientos corporativos, emprendimientos en familia, emprendimientos sociales, emprendimientos habituales, emprendimientos en serie, asimismo, expresa que hay una serie de factores que deben existir para el desarrollo óptimo de esta serie de emprendimientos. 


\section{FACTORES PARA EL DESARROLLO DE LA ACTIVIDAD EMPRENDEDORA EN LA EMPRESA FAMILIAR}

Según Vallmitjana (2011), existen ciertos factores que permiten el desarrollo efectivo de la actividad emprendedora, nombrados a continuación:
a) contexto macroeconómico
b) acceso a fuentes de financiación
c) contexto social
d) contexto tecnológico
e) políticas de soporte de los gobiernos
f) papel de la Universidad
g) las incubadoras

En conclusión, el emprendedor no es el inventor, ni el inversor capitalista. El emprendedor tiene la función de asignar recursos, tomar decisiones y de organizar la actividad económica (Vallmitjana, 2011, siguiendo a Schumpeter 1911).

En síntesis, el emprendimiento es la capacidad que tiene un individuo para visualizar una oportunidad en medio de un contexto favorable o poco favorable, convertirlo en un proyecto innovador; combinando elementos como: motivación, conocimiento, actitud, aptitud y resiliencia, a fin de ejecutarlo y llevarlo a cabo para coadyuvar e intervenir en la transformación social y económica de las sociedades, es decir, al desarrollo de los países, sus regiones y sus comunidades, a través de la creación de nuevas empresas.

Hay toda una gama de factores nombrados anteriormente, que permiten el desarrollo de la actividad emprendedora, y que por ende da origen a la constitución de la empresa familiar, considerándola como un tipo de organización empresarial que cada día cobra más importancia por la complejidad de las relaciones que subyacen en su dinámica (Romero et., al 2015). 
Estos autores señalan, la superposición de roles en los miembros de la familia que ejercen funciones dentro de la empresa, establece que sentimientos o afectos familiares se conjuguen con la toma de decisiones empresariales.

De allí que, este tipo de relaciones tengan una magnitud trascendental, pues la coexistencia de roles como: padre/jefe, hijo/ subordinado, hermano/socio, hacen que las consecuencias derivadas del intercambio entre ambos subsistemas puedan conducirla a la quiebra y/o al distanciamiento del grupo familiar; o en caso contrario, pueda llevarla por el camino del éxito, ya que sus miembros estarán dispuesto a dar lo mejor de sí para la obtención de los mejores resultados económicos que serán transferibles a sus descendientes (Romero et., al 2015).

Rodríguez y Chauca (2013), definen la empresa familiar como aquella dirigida y operada por los miembros de una familia, su evolución y dinámica suele estar asociada con el control accionario o la propiedad en manos de una familia. De igual forma, señalan una empresa familiar puede ser de cualquier dimensión (micro, pequeña, mediana o grande), a las micros y pequeñas empresas (MYPES) familiares debe señalarse que en México, desde junio de 2009, se adoptó una estratificación a partir del número de trabajadores, las ventas anuales y el sector económico

Según Belausteguigoitia (2007), la empresa familiar es una organización controlada y operada por los miembros de una familia. Desde una perspectiva diferente, son empresas con alma dado que el corazón de las familias está en ellas.

Otra definición es la de Leach (1993), que concibe a la empresa como un vínculo familiar. La familia como entidad puede controlar efectivamente las operaciones de la empresa, porque posee más del $50 \%$ de las acciones o porque miembros de la familia ocupan importantes posiciones en la dirección de la empresa.

De acuerdo a Poza (2004) una empresa familiar es aquélla con los siguientes elementos: (a) control sobre la propiedad ( $15 \%$ o más) por parte de dos o más miembros de una familia o asociación de familias, (b) la influencia estratégica de los integrantes de la familia en la dirección de la empresa, ya sea actuando directamente en esos niveles jerárquicos, dando forma continuamente a la cultura 
o fungiendo como asesores o miembro del consejo de administración, (c) interés por las buenas relaciones familiares o sueño de continuidad de generación a generación.

Dentro de este marco, Ginebra (1999) afirma sólo se puede hablar de empresa familiar en todo su sentido cuando: (a) el control accionario es de la familia, pudiendo como máximo comprender los grupos minoritarios ajenos un paquete que no controle nada o muy poco, (b) la familia dirige la empresa, de tal forma que la dirección general y varios otros puestos claves en la estructura ejecutiva son ocupados por miembros de la familia, y (c) los activos en la empresa deben alcanzar al menos un $25 \%$ de los activos patrimoniales globales.

Sin embargo, para que estas empresas tengan un desempeño óptimo y alcancen los objetivos propuestos deben tener un entorno favorable para su creación y sostenibilidad.

Según el Instituto de la Empresa Familiar Española (2014), esta capacidad productiva ha sido reconocida por numerosos gobiernos del mundo, por lo que se han diseñado políticas destinadas al fomento del emprendimiento de negocios familiares, para ello se han creado incentivos que facilitan desde el punto de vista jurídico y administrativo la apertura de estas empresas, así como también se han creado programas de asistencia técnica y financiera dirigidas al sector; destaca además, programas de formación y capacitación de jóvenes líderes empresariales y políticas de carácter fiscal, como la exoneración de impuestos, entre otros muchos mecanismos de incentivos utilizados.

De acuerdo a lo expresado por los autores, se puede afirmar la relevancia que hoy día constituye la contribución que proporcionan este tipo de empresas denominadas familiares a la recuperación económica de los países, ya que este tipo de organizaciones surgen de la innovación y la iniciativa de emprender de los ciudadanos que forman parte de un grupo familiar consanguíneo con el fin de obtener beneficio propio y social.

Aunado a esto, se debe tomar en consideración algunos factores como: la globalización, entorno económico, político y social que puedan afectar el normal desempeño de sus procesos y por ende la sostenibilidad de la empresa familiar. 
A los fines de lograr una empresa familiar sostenible y exitosa, se debe plantear a lo interno según Sukier et al., (2017) la perspectiva y el alcance de una dirección estratégica, en la cual se plantean los enfoques que desde una visión gerencial, impactan en el contexto de las organizaciones. Como componentes relevantes que afianzan el fortalecimiento de las capacidades directivas, se reafirma la gestión estratégica, en función de la complementariedad con los estilos de liderazgo y sus implicaciones la gestión integral del talento humano.

La dirección estratégica, permite promover sus incidencias en la efectividad de la gestión de clientes y mercados, la cual se proyecta desde la definición de procesos para la productividad donde la creación de valor, constituye parte fundamental de los componentes básicos que requieren ser desarrollados desde el contexto de las empresas familiares (Sukier et al., 2017).

Por tal motivo, es necesario, que a lo interno de las empresas familiares se pueda desarrollar y lograr la generación de valor, mediante la estandarización de procesos y la consolidación de la plataforma que prevé la efectividad de la dirección estratégica, mediante los componentes básicos que proyectan la formulación, despliegue, seguimiento y evaluación de la gestión. Este proceso considera direccionar la estrategia, en función de la estructura organizacional, así como también con la cultura para el alcance de los objetivos empresariales (Sukier et al., 2017).

En este sentido, Sukier et al. (2017), esbozan ciertos componentes básico que deben existir para el desarrollo de un a dirección estratégica efectiva, todo ello, en el marco de una gestión integral, se prevé la conceptualización y operatividad los principios básicos que sustentan la efectividad de la gestión de dirección estratégica, desde las empresas familiares del sector se mencionan: a) Competitividad vista desde la capacidad de generar características distintivas al producto, bienes y servicios en función del mercado, b) Innovación y desarrollo basado en la creatividad, emprendimiento por parte de los miembros de la empresa, esta amerita responder a la capacidad que busca fortalecer el sector como estratega de crecimiento y sostenibilidad permanente, c) Autoaprendizaje y mejoramiento continuo, respaldada por la visión integral de las empresas hacia con el entorno y 
su forma de poder enfrentar las transformaciones de mercados en un orden global y local.

Por su parte, North y Baumol (1990) citados en Rodríguez et al., (2017), orienta la sostenibilidad y éxitos de los emprendimiento o empresas familiares al contexto económico y sociocultural, su teoría se fundamenta en el papel determinante de las instituciones, la estructura de incentivos y oportunidades para una sociedad; en consecuencia, las instituciones políticas y económicas son las que determinan el desempeño económico a largo plazo.

Este enfoque define las instituciones formales e informales. Las primeras comprenden las leyes, los reglamentos y los procedimientos gubernamentales, mientras que las instituciones informales incluyen las ideas, las creencias, las actitudes y los valores de las personas, es decir, la cultura de una sociedad determinada. Es así, como los futuros empresarios y sus empresas, como agentes económicos, verán limitadas sus acciones por esta estructura institucional.

Es decir, el entorno institucional de una sociedad determina los beneficios ligados a las distintas oportunidades y dirige la actividad emprendedora hacia esas actividades en las que los beneficios son relativamente más altos. Por ende, la relevancia de una institucionalidad adecuada como requisito indispensable para una positiva interrelación entre el emprendimiento y el crecimiento (North y Baumol 1990 citados en Rodríguez et al., 2017)

En conclusión, se considera relevante contar con un contexto institucional y económico favorable que permita el desarrollo de emprendimientos y empresas familiares, de igual forma, es necesario aplicar a lo interno de las empresas familiares luego de constituidas una dirección estratégica sistémica y evolutiva, basada en el logro de los objetivos propuestos de manera exitosa, y a su vez permita el desarrollo y crecimiento empresarial consolidado, y por ende la efectividad esperada que conlleva a la sostenibilidad de la empresa familiar.

Por otro lado, Romero et al., (2015), afirman, uno de los temas álgidos en el crecimiento, la sostenibilidad y el éxito de una empresa familiar, es plantearse el camino hacia la internacionalización, la cual es abordada usualmente por la vía de la exportación de bienes y servicios. 


\section{FACTORES QUE AFECTAN LA INTERNACIONALIZACIÓN DE EMPRESAS} FAMILIARES

Según Romero et al., (2015), el proceso de internacionalización empresarial para un país conlleva muchas ventajas, especialmente cuando se trata de operaciones de exportación, pues representa una ampliación del mercado que brinda extensas posibilidades de lograr mayores escalas de producción que se revierten en un incremento del empleo y el ingreso nacional. En tal sentido, a la hora de evaluar la conveniencia o no de iniciar tal proceso, se requiere que el empresarioemprendedor- considere qué factores inciden en su desempeño exportador.

Dentro de esta perspectiva, Romero et al., (2015) señalan los factores de carácter interno, siguiendo a Francés y García (1998), son aquellos recursos y capacidades que posee una organización y que determinan sus ventajas competitivas, estos aspectos están bajo control de la empresa, de los cuales cabe mencionar: recursos financieros, tecnológicos, productivos, humanos, organizativos, así como también se incluye la actitud del empresario frente al proceso exportador, por nombrar algunos.

Romero et al., (2015), concluyen en un estudio realizado a empresas familiares en la región zuliana-Maracaibo-Venezuela, que el problema radica en que la estructura de costos no permite ser competitivos internacionalmente, pues las exportaciones se han encarecido producto de la aplicación de la política cambiaria, ya que la fijación de una tasa de cambio fija en bolívares por dólar (sin poderla ajustar a los constantes aumentos en los costos internos) hace de la actividad exportadora un negocio poco rentable. Adicionalmente, no hay divisas para el sector, la inseguridad jurídica es enorme, persiste el retraso en la emisión de certificados. Aunado al desplazamiento de nuestros rubros debido al surgimiento de otros competidores a nivel mundial, más seguros y confiables que Venezuela (León, 2009 citado por Romero et al., 2015).

Estos autores afirman en su estudio, que el entorno es captado de manera hostil, haciendo que las decisiones en el área exportadora dependan en gran medida de las perspectivas de evolución de éste. Fundamentalmente, en el ámbito 
nacional y local los factores concernientes al espacio político, jurídico y económico reflejan un grado elevado de incertidumbre en cuanto a su dirección futura, por lo que la dinámica internacional descansa en la evaluación de dichos aspectos, pasando incluso por encima de una valoración positiva del mercado mundial que se sustenta en la explotación de las ventajas (competitivas y comparativas) que presentaron las unidades económicas investigadas (Romero et al., 2015).

Lo anteriormente expresado por los autores, permite afirmar que la internacionalización de las empresas familiares constituye una estrategia de expansión, crecimiento, éxito y sostenibilidad, al mismo tiempo se deben tomar en consideración las ventajas y capacidades competitivas con que cuenta la empresa familiar, que a lo interno y a nivel nacional puedan resultar favorables, esto, sin dejar de lado el contexto entorno no manejado por ellas, como lo son los aspectos políticos, económicos, fiscales y tributarios constituyendo un panorama poco favorable que impidan la intención hacia la internacionalización.

\section{REFLEXIONES FINALES}

La reflexión teórica realizada, en el presente trabajo de investigación colocó en manifiesto el papel relevante que hoy día representan el Emprendimiento y la Innovación como agentes potenciadores para la creación de las empresas familiares, esta triada, representa un factor clave para la recuperación y el desarrollo de las economías a nivel mundial, constituyendo el $90 \%$ de la actividad productiva y el aumento en gran medida del PIB de los países (Poza, 2004; y Rodríguez y Chauca y 2013).

De igual forma, constata la imperante necesidad de fortalecer la innovación como factor relevante para el emprendimiento, mediante el establecimiento por parte de los gobiernos de los países del mundo de una mayor inversión hacia la investigación y el desarrollo ( $(+D)$, en universidades, empresas, fundaciones; de igual forma, se deben fortalecer los sistemas de innovación, todo ello para coadyuvar en el aumento de la productividad mediante creación de nuevos productos o bienes y servicios. 
Desde la perspectiva del emprendimiento, se confirma el papel fundamental y potenciador para impulsar la ejecución de proyectos innovadores que coadyuvan a la creación de las empresas familiares, basadas en la consanguinidad de un grupo de personas y generadoras de diversas fuerzas de trabajo que contribuyen al desarrollo económico a nivel mundial, sus regiones y comunidades, como se expresa a lo largo del estudio se considera la principal fuente de ingreso para la economía, proveyendo el bienestar y la transformación social.

Sin embargo, es necesario promover la sostenibilidad de este tipo de empresas; mediante estrategias como la dirección estratégica de la empresa familiar, tomando en cuenta el logro de los objetivos y la generación de valores, de igual forma se debe tomar en consideración las capacidades internas de la empresa y tomar en cuenta el entorno, todo ello, estableciendo una plataforma sistémica que permitan la eficiencia en los procesos, a través de la innovación, emprendimiento y el autoaprendizaje.

Otro aspecto importante, lo constituye la aplicación de un sistema institucional adecuado orientado a esfuerzos en función de políticas públicas definidas hacia el fomento de la actividad emprendedora; estas proveen los recursos financieros que deben ser aprovechados para el desarrollo tanto de las empresas familiares como de las comunidades en las cuales se insertan que permitan el crecimiento de las economías.

De igual forma, se considera la internacionalización como una estrategia de sostenibilidad y expansión, proveyendo un sistema de exportaciones que faciliten dicha internacionalización; ya que mediante ella se proveen los recursos financieros que deben ser aprovechados para el desarrollo tanto de las empresas familiares como de las comunidades en las cuales se insertan, y por ende, colaboran a los ingresos estatales, y el incremento productivo de los países.

Lo anterior, estipula la existencia de una cultura emprendedora apoyada en la innovación que permita una constante actualización potenciar la economía de los países, esta afirmación se hace, ya que en la actualidad todavía existen países que dan poco o nulo valor a la innovación y el emprendimiento, debido a la resistencia al cambio, el miedo al fracaso y al desgano de innovar por parte de la población. 
En este sentido, esta cultura emprendedora de la que hablamos debe provenir de la articulación de todos los sectores -sociedad, sector público y privado, sector bancario, universidades; mediante iniciativas de involucramiento con las comunidades, valoración de sus ideas, proporcionando un entorno político y económico que permita el desarrollo de emprendimientos- proyectos innovadores, considerados como nuevas empresas de carácter familiar, exitosas, eficientes y sostenibles que coadyuven a su beneficio personal y el de las sociedades del mundo y sus economías.

\section{REFERENCIAS BIBLIOGRÁFICAS}

Belausteguigoitia Rius, Imanol (2007), Empresas familiares: su dinámica, equilibrio y consolidación. Editorial McGraw Hill Interamericana. México.

CORFO (Corporación de Fomento de la Producción) (2014). Perfil del emprendedor chileno y sus emprendimientos. Extraído de: http://repositoriodigital.corfo.cl/bitstream/handle/11373/8399/PERFIL\%20DE \%20EMPRENDEDORES.pdf?sequence=1 Consulta: 19-08-2018.

Diccionario de la Academia Real Española (2001). Emprendimiento. Extraído de: http://buscon.rae.es/drael/SrvltConsulta?TIPO BUS=3\&LEMA=cultura. Consulta: 18-08-2018.

Drucker, Peter (1985). La innovación y el empresario innovador. Editorial Ed Edhasa. España.

Ginebra, Joan (1999), Las empresas familiares: su dirección y continuidad, Panorama. México.

Gort, Michael y Steven Klepper (1982). Time Paths in the Diffusion of Product Innovations. Economic Journal, vol. 92, № 367. Gran Bretaña (Pp. 2-25).

Instituto de la Empresa Familiar (2014). La Empresa Familiar: Cifras. (España). Extraído de: http://www.iefamiliar.com/cifras/1. Consulta: 03/08/2018.

Leach, Peter (1993), La empresa familiar. Granica - Vergara. Argentina.

Navarro, Lucas y Villegas, José (2016). Innovación y emprendimiento en Chile: diagnóstico y políticas. En: La Fuerza de la Innovación y el Emprendimiento ¿Es probable que Latinoamérica se suba al carro de las sociedades del conocimiento? CONICYT (Comisión Nacional de Investigación Científica y Tecnológica). Chile.

Oporto Henry (2016). Innovar para crecer Repensando el modelo de crecimiento en Bolivia. En: La Fuerza de la Innovación y el Emprendimiento ¿Es probable que Latinoamérica se suba al carro de las sociedades del conocimiento? CONICYT (Comisión Nacional de Investigación Científica y Tecnológica) (2016). Chile.

Poza, Ernesto J. (2004), Empresas familiares. Thompson. México.

Romero Borré, Jenny; Hernández Fernández, Lissette; Bracho Rincón, Neida. (2015). Incidencia del entorno en las exportaciones de las empresas 
familiares del estado Zulia- Venezuela. Opción, vol. 31, núm. 77, mayoagosto, 2015. ). Venezuela (Pp. 126-144).

Rodríguez, Anuar y Chauca, Pablo Manuel (2013). Problemática y alternativas de financiamiento para las micros y pequeñas empresas familiares de Michoacán: estudio de caso. INCEPTUM, Vol. VIII, No. 15. Julio - Diciembre, 2013, México, (Pp. 127 - 158).

Rockefeller, David. (2004). Gestión Efectiva de Emprendimientos Sociales: Lecciones Extraídas de Empresas y Organizaciones de la Sociedades Civil en Iberoamérica, Extraído de: https://books.google.co.ve/books?id=SU75nsEP9MMC\&pg=PA24\&dq=empr endimiento+social\&hl=es\&sa=X\&redir esc=y\#v=onepage\&q\&f=false. Consulta: 20/08/2018.

Schumpeter, Joseph Alois. (1942). Capitalism, Socialism, and Democracy: Harper \& Brothers. United States.

Sukier, Harold Bernardo; Molina Neira; Portillo Rafael; Hernández Lissette; Fábregas Carlos (2017). Dirección estratégica en las empresas familiares. Revista Espacios. VOL. 38 NN$^{\circ}$.24.México (Pp.2-23).

Vallmitjana, Nuria (2011). La actividad emprendedora de los graduados IQS", que tuvo como objetivo la evaluación del emprendimiento de los graduados IQS. Tesis Doctoral, Departamento de Gestión Empresarial. Universidad Ramón Liull. España.

Vesga, Rafael (2017). Emprendimiento e Innovación en Colombia: ¿qué nos está haciendo falta?

de: https://web.unillanos.edu.co/docus/Emprendimiento\%20e\%20innovacion.pdf Consulta: 23/08/2018. 